\title{
Intervención didáctica para promover el aprendizaje de las matemáticas, en niños con discalculia
}

\author{
Andrea Carolina Rojas Suarez ${ }^{1}$ Adriana Patricia Contreras Hernández ${ }^{1}$ Mayra Alejandra Arévalo Duarte ${ }^{2}$
}

Recibido:

Abril 23 de 2011

Aceptado:

Diciembre 2 de 2011
${ }^{1}$ Licenciada en Matemáticas e Informática UFPS

\section{Resumen}

Este estudio tiene como propósito favorecer el aprendizaje de las matemáticas en niños con discalculia mediante el desarrollo de estrategias didácticas. La metodología investigativa se enmarca dentro del paradigma cualitativo, con un método etnográfico de tipo exploratorio. Su objeto de estudio requiere ser abordado a partir del registro de observaciones del desenvolvimiento de los estudiantes con dificultades en el aprendizaje de las matemáticas a través del desarrollo de pruebas en esta área. Las categorías abordadas corresponden a factores que intervienen en el aprendizaje, las alternativas de enseñanza, el aprendizaje de las matemáticas en niños con discalculia y actividades matemáticas de acuerdo al pensamiento numérico, las cuales marcaron la ruta de ejecución para abordar el objeto de investigación. Finalmente, se concluye que las estrategias utilizadas permitieron al estudiante no sólo conocer el tema a partir del proceso desarrollado sino al mismo tiempo reforzar y mantener constante su motivación y participación en el aprendizaje de esta área fundamental en el currículo escolar.

Palabras clave: Aprendizaje, discalculia, estrategias didácticas, pensamiento numérico, paradigma cualitativo.

\section{Abstract}

This study was aimed to encourage the learning of mathematics in children with developmental dyscalculia through education and teaching strategies. The research methodology is framed within the qualitative paradigm, with an exploratory ethnographic method, as its object of study needs to be addressed from the observational record of the development of students with learning difficulties in mathematics through the development of evidence in this area. The categories addressed relate to factors that affect learning, alternative teaching, learning mathematics in children with dyscalculia and mathematics activities according to numerical thinking, which marked the path of execution to address the subject of investigation. Finally, we conclude that the strategies used allowed the student not only know the subject from the process developed but at the same time, enhance 
and maintain constant motivation and participation in learning in this key area in the school curriculum.

Keywords: Learning, dyscalculia, teaching strategies, numerical thinking, qualitative paradigm.

\section{Introducción}

A lo largo de la vida escolar, algunos estudiantes muestran necesidades específicas en su proceso de aprendizaje: requieren una explicación adicional en relación con algún contenido, demandan nuevos procesos como consecuencia de la rapidez en la consecución de las metas establecidas en comparación con el grupo en general, manifiestan dificultad en la adquisición y uso de la lectura, escritura, razonamiento lógico, habilidades matemáticas o demuestran intereses significativamente diferentes al grupo. Estos estudiantes, generalmente, son considerados diferentes en el aula de clase y las soluciones dadas, por parte de sus maestros, han sido, en algunas ocasiones, la remisión a los servicios especiales ajenos al sistema escolar como consecuencia de un diagnóstico realizado con escasa profundidad educativa, o en los mejores casos, la modificación ligera de la metodología de trabajo en el aula de clase sin ningún sentido, ni objetivo de aprendizaje.

En la actualidad y ante este panorama, el sistema educativo ha planteado cambios significativos tanto en las prácticas docentes habituales como en la concepción y diseño de los servicios educativos. Para los dos casos, las escuelas deben aunar esfuerzos y prepararse para asumir el reto de atender a estudiantes que demanden una dificultad especial, y para asumir la profunda transformación que exige la sociedad actual. A partir de esta necesidad, el nuevo concepto de "dificultades de aprendizaje" cambia de forma considerable, recogiendo nuevas expectativas y nuevas prácticas docentes que favorecen la inclusión de los niños, especialmente los que presentan este tipo de necesidades, aclarando, que no se trata de impedimentos visuales, auditivos, retraso mental u otros que requieran unas condiciones educativas especializadas. Por consiguiente, el maestro debe tener la capacidad de atender estas dificultades de forma natural, cálida y con una pedagogía, que permita el desenvolvimiento normal de la vida escolar, sin necesidad de retrasar el proceso de aprendizaje de los niños. Frente a estas circunstancias, el maestro debe reflexionar y determinar los aspectos específicos que propicien el proceso de aprendizaje, cuestionando los factores que intervienen en su práctica pedagógica.

El presente estudio, inscrito en la línea de investigación de "enseñanza de las matemáticas", plantea como pregunta central determinar ¿Por qué es importante utilizar estrategias didácticas que faciliten el aprendizaje de las matemáticas en niños con problemas de discalculia? Y tiene como propósito, favorecer el aprendizaje de las matemáticas en niños con problemas de discalculia mediante el desarrollo de estrategias pedagógicas y didácticas, contribuyendo a desarrollar el pensamiento complejo a partir del diseño y resolución de problemas, aplicación de conceptos y habilidades matemáticas en la toma de decisiones y evaluación de los procesos realizados para aprender de estos. El desarrollo y fortalecimiento de estos procesos son fundamentales en el caso de los niños con dificultades en el aprendizaje de las matemáticas (DAM), especialmente en los 
niños con problemas de discalculia, ya que sus dificultades aumentan cuando las actividades están enfocadas al desarrollo de procesos que requieren de pensamiento complejo.

Es importante que los docentes tengan presente que las matemáticas son consideradas habitualmente "difíciles" por los estudiantes de cualquier nivel educativo. Razón por la cual Brunner (1995) propone que el aprendizaje de conceptos matemáticos se introduzca a partir de actividades simples, que los estudiantes puedan manipular para descubrir sus principios y la posibilidad de soluciones. Según este autor, la enseñanza de la matemática promueve que se trabaje con objetos concretos antes de pasar a realizar procesos de abstracción. En el nivel de primaria, la matemática escolar ha de realizarse de modo que los alumnos se apropien de los conocimientos esenciales y desarrollen las habilidades que les permitan aplicar de forma independiente sus conocimientos para resolver los problemas del entorno social. Es así como la presente investigación expone la necesidad de diseñar o adecuar actividades y recursos didácticos que vayan de la mano con una metodología de enseñanza y aprendizaje para la búsqueda del favorecimiento de experiencias significativas en el aprendizaje del área.

En el marco de los referentes teóricos, se establece la necesidad de caracterizar a los niños con problemas de discalculia y establecer las posibles causas para determinar las formas de intervención pedagógica y didáctica que se puede realizar desde la escuela.

En cuanto al problema de discalculia o trastorno del cálculo (American Psychiatric Association -APA, 1987), se tiene que este término hace referencia a un problema de tipo cognoscitivo en la niñez que afecta la adquisición normal de las habilidades matemáticas. Según Vázquez, (2007), siempre que un niño con capacidades intelectuales normales presenta una dificultad específica en el proceso de aprendizaje de las matemáticas se le atribuye la culpa a la "dificultad que conlleva la materia" o simplemente a que "no se le enseña bien". Sin embargo, al igual que hay un trastorno del aprendizaje relacionado con la lectura denominado dislexia, también se da un trastorno del aprendizaje que incide en el rendimiento de las capacidades de cálculo aritmético: la discalculia. Una disfunción que afecta a un porcentaje de la población infantil (entre el 3\% y el 6\%). La edad para detectar un problema de discalculia está entre los seis y los ocho años de edad, momento en que se introducen las matemáticas como materia independiente y se puede comparar el rendimiento de unos niños con otros.

Según Coll (1993), hablar de niños y jóvenes con problemas de discalculia es considerar a un grupo heterogéneo que presenta una característica común: "bajo rendimiento en matemáticas", pero que difieren en las causas. Algunas variables asociadas a estas causas son: dificultad para concentrarse en clase, impulsividad, problemas de autoestima, de motivación, malos hábitos de estudio, preocupación por bajo rendimiento, cambio de escuela, rechazo por parte de los compañeros, factores familiares o cualquier otra preocupación en particular. Sin embargo, Alarcón et al. (1997), afirma que los problemas de discalculia también obedecen a un fuerte componente genético con el que se combinan además factores ambientales.

De acuerdo a lo anterior, se establece que la metodología didáctica pertinente para la atención de los niños con problemas de aprendizaje gira alrededor del concepto de "adaptación curricular", que consiste en ajustar el programa educativo común a las posibilidades y necesidades que demande el estudiante, es decir, se deben identificar las fortalezas, debilidades y necesidades educativas de los estudiantes para planear

Diciembre 2011 ISSN 0122-820X y organizar el currículo: contenidos, metodología, estrategias y sobre todo los procesos de evaluación.

Los currículos inclusivos se construyen de manera flexible no sólo para permitir su adaptación y desarrollo a nivel de la escuela. Sino también para permitir adaptaciones y modificaciones 
para responder a las necesidades individuales de los estudiantes y a los estilos de trabajo de los maestros (...) UNESCO (2004:110)

Este marco teórico nos proporciona una metodología que comprende tres fases, las cuales definen los elementos que son fundamentales para realizar las adaptaciones curriculares pertinentes que garantizan el acceso de los estudiantes al currículo. Primera fase: relacionada con la recolección de información del estudiante: estilos y estrategias de aprendizaje, motivación, competencias, entre otros. Segunda fase: relacionada con la recolección de información del contexto: organización grupal, actividades de aprendizaje, comunicación, estructura, reglas de interacción, normas, comportamientos, entre otros. Y finalmente, la Tercera fase: relacionada con la interpretación de la información. El cumplimiento de estas fases proporciona la información necesaria para plantear la metodología, las estrategias pedagógicas y didácticas y, sobre todo, el proceso evaluativo pertinente para trabajar con los estudiantes que demanden este tipo de atención.

A partir de estas metodologías, se establecen unas series de estrategias de apoyo que incluyen diferentes tipos de recursos que contribuyen a que la resolución de la tarea se lleve a buen término (González y Tourón, 1992). Este tipo de estrategias tienen como finalidad sensibilizar al estudiante con lo que va a aprender, integrando tres aspectos: la motivación, las actitudes y el afecto (Beltrán, 1993). Estos aspectos determinan en gran medida las habilidades que pondrán a prueba los estudiantes en las tareas de aprendizaje particular. El uso de material didáctico es fundamental para la enseñanza de todas las áreas, sin embargo, debe ser incorporado en el momento adecuado y con una serie de finalidades. Según Parcerisa (1999), estas finalidades deben ser:

- Aproximar al alumno a la realidad de lo que se quiere enseñar, ofreciéndole una noción más exacta de los hechos o fenómenos estudiados.

- Motivar la clase durante todo el tiempo.

- Facilitar la percepción y la comprensión de los hechos y de los conceptos

- Dar oportunidad a que se manifiesten sus aptitudes y se desarrollen las habilidades específicas.

Abordar la enseñanza de las matemáticas a través del uso de material didáctico se justifica, puesto que hace posible el planteamiento de situaciones significativas y el aprendizaje a través de la solución de problemas.

El uso del material didáctico debe estar inmerso en el planteamiento y la solución de problemas o de situaciones didácticas que den oportunidad a los estudiantes, no sólo de indagar personalmente sobre posibles soluciones, sino también de expresarlas y razonar su validez.

\section{Materiales y métodos}

De acuerdo a la naturaleza del objeto de este trabajo, la investigación realizada reúne las características de un estudio cualitativo, de tipo exploratorio y etnográfico. Su propósito es estudiar los hechos tal como ocurren en el contexto y registrar a partir de observaciones, lo que sucede en cada una de las intervenciones, haciendo uso de una variedad de instrumentos que permiten registrar la información que se obtiene a partir de este método. Asimismo, se pretende establecer prioridades para investigaciones posteriores o sugerir afirmaciones que puedan ser verificables.

La población de estudio corresponde a los estudiantes de cuarto grado del Colegio Municipal Gremios Unidos de la ciudad de Cúcuta, Norte de Santander, seleccionada por tener el grado intermedio de primaria, donde se destacan con mayor 
frecuencia las dificultades en el aprendizaje de las matemáticas (DAM). La muestra corresponde a 17 estudiantes que, según el maestro y el registro académico, presentan mayores dificultades en el aprendizaje de las matemáticas. Estos estudiantes fueron seleccionados intencionalmente para destacar casos característicos de la población objeto de estudio. A continuación se describen las etapas de desarrollo de la investigación:

Etapa inicial: en la primera fase se aplicó el instrumento "ficha personal", el cual permitió registrar los datos personales, los aspectos importantes del factor familiar, social y escolar. Y el segundo instrumento "diagnóstico inicial", que permitió recolectar información sobre los conocimientos previos de los estudiantes.

Etapa intermedia: en la segunda fase se aplicaron actividades escritas y didácticas de la siguiente forma: en un día se hizo la actividad escrita correspondiente a las fichas y al día siguiente se realizaron una o más actividades didácticas que complementaron y reforzaron el tema. Estas actividades, registradas en el diario de campo, permitieron recolectar las dificultades sobre los contenidos manejados y, al mismo tiempo, determinar la importancia de aspectos tales como la motivación, el comportamiento y los intereses de los estudiantes.

Etapa final: para finalizar el proceso, en la tercera fase se realizaron visitas familiares a los estudiantes del sector más cercano a la institución, con el fin de verificar y complementar la información proporcionada.

Instrumentos utilizados y actividades desarrolladas en la intervención:

Tabla 1. Instrumentos aplicados

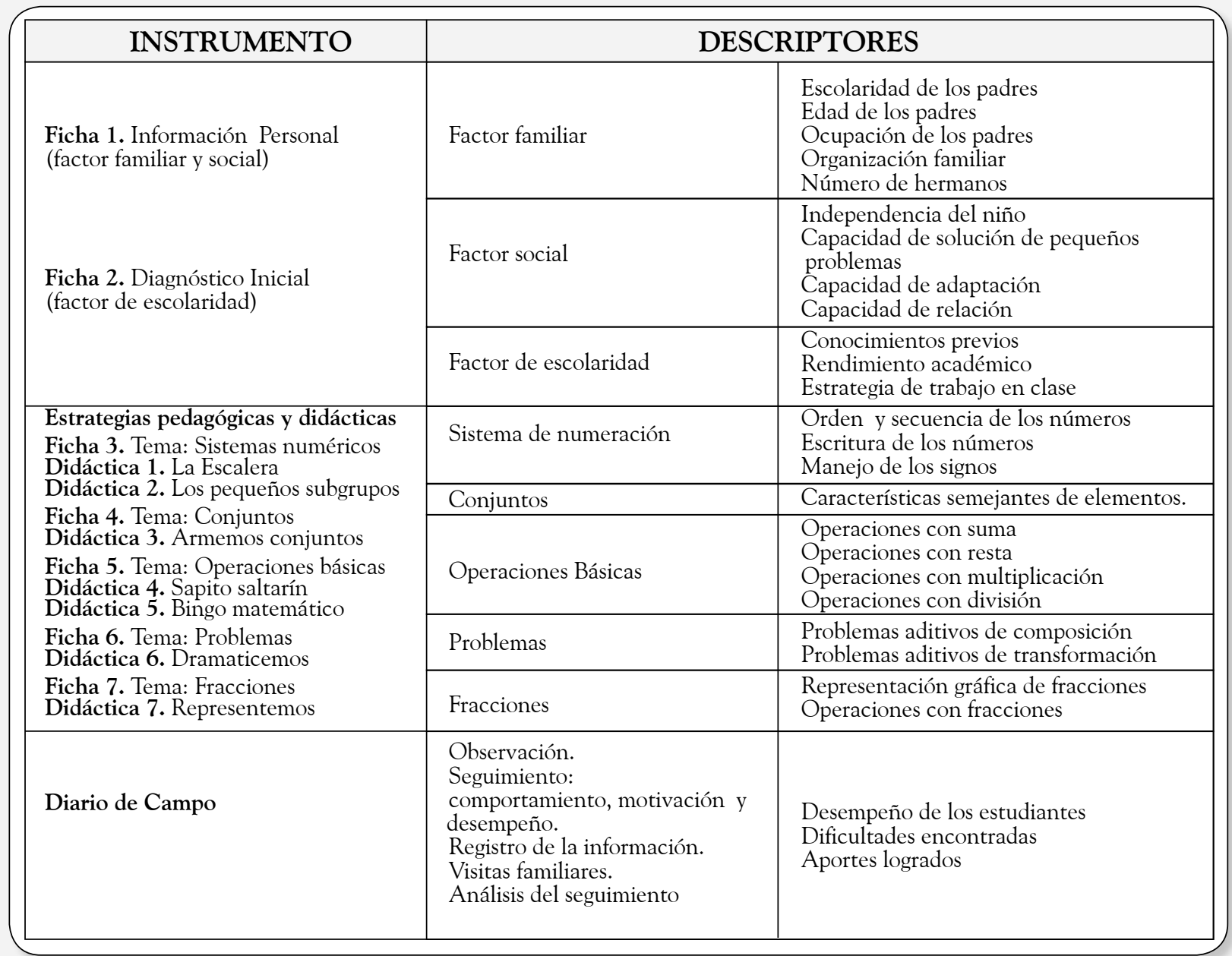




\section{Resultados y discusión}

En cuanto a la identificación de las dificultades que presentan los niños con el aprendizaje de las matemáticas, de acuerdo con el factor familiar y social, se encontró que una de las posibles causas puede estar en la constitución y estabilidad de la familia y en el papel que juegan los padres en el acompañamiento académico de sus hijos. Coll (1993), contribuye afirmando que cuando el menor está triste, baja de inmediato su rendimiento porque la atención está centrada en sus preocupaciones. Por lo general, esto ocurre por una reacción a ciertas circunstancias que afectan directamente al niño o joven, como la separación de los padres, la muerte de algún familiar, el rechazo de los compañeros o cualquier otra situación en particular.

Factores familiares. Los niños seleccionados pertenecen al mismo estrato socioeconómico. El nivel educativo de la mayoría de los padres es de educación básica primaria y secundaria, el $15 \%$ de esta población no tienen ningún tipo de escolaridad. El 80 \% de los niños conviven sólo con uno de sus padres y con otros familiares y el número de hermanos en casi todos los casos es mayor de dos.

E14. En la ficha personal respondió a: ¿Qué nivel de educación poseen sus padres? "El nivel de escolaridad de mis padres es de básica primaria".

E1. En la ficha personal respondió a: ¿Con quién vive? "Vivo con mi mamá y mi abuela".

E5. En la ficha personal respondió a: ¿Cuántos hermanos tiene? "tengo cinco hermanos, dos hombres y tres mujeres".

Factores escolares y sociales. A la mayoría de los niños se les dificulta adaptarse al ambiente escolar, son dependientes, se distraen fácilmente, necesitan varias explicaciones para comprender algún tema, se les dificulta relacionarse con los demás y, al mismo tiempo, los conocimientos previos en el área son muy limitados. Según Alarcón et al., (1997), los niños y jóvenes que presentan estas características son personas que, sin duda alguna, tienen problemas de aprendizaje.
E6. En la ficha personal respondió a: iEs sociable? iSe relaciona fácilmente? "No me gusta relacionarme y soy muy poco sociable".

E1. En la visita al hogar, a la siguiente pregunta, respondió: iTiene capacidad de adaptación a cualquier situación? "Si, se me dificulta adaptarme a los cambios".

E13. En la ficha personal respondió a: iHa repetido cursos? "Si, repetí tercero de primaria por mal rendimiento académico".

En cuanto a las dificultades presentadas por los estudiantes en las actividades matemáticas planeadas de acuerdo al pensamiento numérico, se comprobó que: estos niños inicialmente mostraron dificultades en la escritura de los números, en agrupar objetos según sus características, en realizar operaciones básicas, en resolver problemas simples y en representar fracciones. Generalmente, un niño con problemas de discalculia presenta este tipo de dificultades, que se reflejan en los errores al leer o al escribir, Luria (1976). Por ejemplo, en el diagnóstico inicial respecto al dominio de los temas:

E1. Maneja bien el signo "menor que" y "mayor que", estableció correctamente los elementos en los conjuntos, y se le facilitó el desarrollo de operaciones básicas. Presenta dificultad en la resolución de problemas y la representación gráfica de fracciones.

E5. Presenta dificultad en el tema de representación de fracciones impropias.

E10. Se le dificulta el manejo del signo "menor que" y "mayor que", igualmente la resolución de problemas y la representación gráfica de fracciones.

En las actividades didácticas 1 y 2 (Ver Tabla 1. Instrumentos aplicados) se evidencia en:

E3. Se le dificulta identificar la operación donde se obtiene un número mayor sin realizar los cálculos. No realiza el ejercicio de completar el número que falta con operaciones correspondientes a la multiplicación y la resta, también tuvo falencias en seguir una serie de suma y multiplicación. 
E11. No identifica la operación donde se obtiene un número mayor sin realizar los cálculos. Realiza incorrectamente el ejercicio de completar el número que falta con operaciones correspondientes a la suma, multiplicación y resta, también tuvo falencias en seguir una serie de suma y multiplicación.

En la actividad didáctica 3 (Ver Tabla 1. Instrumentos aplicados) se evidencia en:

E4. Solicitaba que se le explicara cada uno de los problemas una o dos veces si era necesario.

E13. No mostró interés por trabajar en los problemas y por consiguiente no realizó ninguno de los ejercicios a desarrollar

En la actividad didáctica 4 y 5 (Ver Tabla 1. Instrumentos aplicados) se evidencia en:

E1. No interpreta el enunciado del problema. Desarrolla las actividades en un lapso de tiempo largo.

E9. Aunque se le dificulta operar con fracciones en suma y resta, efectuó correctamente la relación de la fracción con la representación gráfica.

Para atender a estas necesidades se planearon, organizaron y desarrollaron estrategias didácticas (Ver Tabla 1. Instrumentos aplicados), en las que se pudo observar las siguientes características:

- Los niños presentaron mejor dominio e interés en las actividades didácticas. El diseño del material permitió familiarizarse con el tema a partir de objetos y objetivos concretos, propiciando la oportunidad de enriquecer la experiencia del estudiante mediante la aproximación a la realidad y ofreciéndole la ocasión para actuar, Parcerisa (1999).

- El contextualizar los problemas a la vida cotidiana permitió a los niños facilitar el entendimiento de las situaciones problemas. Un ejemplo visible fue la dramatización del tema "problemas". Estas experiencias significativas aproximaron al estudiante a la realidad de lo que se proponía enseñar, Parcerisa (1999).

- Los niños aumentaron su nivel de seguridad y confianza antes y durante el desarrollo de las actividades (Ver Tabla 1. Instrumentos aplicados. Ficha 3 a 7), cuando se les mostraban sus errores y se les presentaban como algo positivo que permitía la corrección y el refuerzo y no la mala calificación.

- Los niños mejoraron sus resultados en cuanto a la solución de problemas con operaciones aritméticas básicas. También aprendieron a realizar operaciones mentales sencillas, a clasificar elementos, a realizar series de números y a comprender mejor los enunciados de los problemas matemáticos.

- Mejoraron su motivación e interés por el área, aclararon dudas, mostraron mayor responsabilidad con sus deberes y aprendieron a socializar e integrase como grupo. En este caso, las estrategias didácticas fueron un elemento indispensable para lograr la motivación de los estudiantes, Beltrán (1993).

\section{Conclusiones}

Para identificar las dificultades en el aprendizaje que presentan los niños con discalculia es de vital importancia tener en cuenta factores como el familiar, social y el escolar. Estos tienen gran influencia en el desempeño de los estudiantes en las áreas específicas y en las relaciones sociales que se tejen en la escuela. Frente a esto, se pudo deducir que los niños de la muestra conviven en un ambiente familiar que brinda poca estimulación cognoscitiva y afectiva y que 
no proporciona estímulos suficientes para la formación de hábitos e intereses sociales y académicos.

Cabe resaltar que los niños con discalculia poseen un coeficiente de inteligencia normal, pero realizan deficientemente una o más operaciones matemáticas (Ver Tabla 1. Instrumentos aplicados: Estrategias pedagógicas y didácticas). Regularmente el niño no aprende al mismo ritmo del grupo, mantiene una conducta que molesta $\mathrm{e}$ interfiere en la disciplina escolar y se muestra aislado; es por esto que necesita recorrer un camino académico más largo que los demás. Para trabajar con estos niños no es aconsejable realizar una caracterización y utilizar estos resultados para desarrollar actividades académicas generales, pues es tratar de elaborar un patrón universal de sus características sin tener en cuenta que cada niño con estas características constituye un caso diferente y debe ser abordado según su necesidad.

En el desarrollo de esta investigación se ha concluido que los docentes, a pesar de utilizar estrategias ajustadas a los proyectos institucionales y normativas dadas por el Ministerio de Educación Nacional -MEN, continúan integrando técnicas tradicionales a estas estrategias como el "dictado y la transcripción", el uso de un libro determinado para el desarrollo de contenidos, el manejo de material sin sentido, sin tener en cuenta las características propias de cada estudiante $y$, en algunas oportunidades, sin seguir la planificación realizada para el desarrollo de es necesario que los docentes planeen y desarrollen estrategias tendientes a alcanzar una enseñanza de calidad contemplando las necesidades y características de sus estudiantes y del contexto actual, de lo contrario, esta situación ira tomando cada vez más fuerza y se convertirá en un problema de gran magnitud.

Se considera que la metodología utilizada contribuye satisfactoriamente al progreso académico de los estudiantes, puesto que se favorecieron procesos como el refuerzo de

conceptos matemáticos, el aumento en la participación, la motivación y el interés por el trabajo en clase, logrando una mayor visión y desenvolvimiento de los estudiantes ante situaciones nuevas que les demandan esfuerzo, responsabilidad y habilidades matemáticas para su desarrollo.

También se pude deducir que los niños con problemas de discalculia muestran mejor rendimiento cuando se trabaja de forma cooperativa, puesto que esta es la metodología de trabajo más acorde a sus características. Realizar un trabajo cooperativo, didáctico y orientado a su cotidianidad permite que se establezcan relaciones sociales y de colaboración donde se pierde la timidez, la desmotivación, la distracción, el miedo y se genera un ambiente de participación, curiosidad, duda, interrogantes ante cualquier situación problema, donde se puede percibir la matemática como un saber en constante construcción a partir de su realidad.

Frente a la pregunta investigativa ¿Por qué es importante utilizar estrategias didácticas que faciliten el aprendizaje de las matemáticas en niños con problemas de discalculia?, se puede afirmar que estas actividades además de favorecer el aprendizaje de los niños con este tipo de características, aumenta su motivación e interés en el área y, también, fortalece la labor del docente que busca enriquecer su práctica pedagógica con actividades que promueven el encuentro docente - estudiante desde su saber disciplinar, en torno a las características del contexto y de sus estudiantes.

La información proporcionada en esta investigación se convierte en un soporte para toda la comunidad educativa: docentes, padres de familia y personas que conviven con niños que presentan dificultades en el aprendizaje de las matemáticas, ya que sus resultados brindan un soporte y una orientación para atender sus necesidades académicas y para construir los lineamientos de una propuesta pedagógica y didáctica que tenga como propósito favorecer el aprendizaje mediante este tipo de metodologías. 


\section{Agradecimientos}

Al Colegio Municipal Gremios Unidos (Cúcuta, N.S.) por permitir y facilitar los espacios y el recurso humano para el desarrollo de esta investigación.

\section{Referencias bibliográficas}

\section{ALARCÓN M., KNOPIK V.S, De FRIES} J.C (1997). Comorbidity of mathematics and reading deficits: evidence for a genetic etiology. USA: Institute for Behavioral Genetics, University of Colorado. Disponible en: http://www.springerlink.com/content/ m84p47j71165171x/. Fecha de consulta: Enero de 2011).

American Psychiatric Association (1987). Diagnostic and Statistical Manual of Mental Disorders. 3ed. Washington, DC: APA.

BELTRÁN, J. (1993). Procesos, estrategias y técnicas de aprendizaje. Madrid, España: Síntesis.

BRUNNER, J. J. (1995). Informe de la Comisión Nacional para la modernización de la Educación. Santiago: Editorial Universitaria.

COLL, César (1993). Aprendizaje escolar y construcción del conocimiento. Buenos Aires, Argentina: Paidós.

GONZÁlEZ, M. C. y TOURÓN, J. (1992). Autoconcepto y rendimiento académico. Sus implicaciones en la motivación y en la autorregulación del aprendizaje. Pamplona, España: EUNSA.

LURIA, A. R. (1976). Basic Problems in Neurolinguistics. New York: Mouton in The Hague.

PARCERISA, Artur (1999). Materiales curriculares: cómo elaborarlos, seleccionarlos y usarlos. Barcelona, España: Graó.
UNESCO (2004). Temario Abierto sobre Educación Inclusiva. Santiago de Chile: UNESCO.

VÁZQUEZ R, M. (2007). Discalculia, la dislexia de los números. [Documento en línea]. Disponible en: http://www.consumer.es/web/ es/educacion/escolar/2007/12/10/172676. php. Fecha de consulta: Enero de 2011. 